\title{
Value of MRI enhanced FLAIR sequence examination combined with CSF TNF- $\alpha$ detection in the early diagnosis of neonatal purulent meningitis
}

\author{
YANLI ZHANG $^{1 *}$, XIAOLIN SUN ${ }^{2 *}, \mathrm{JUN} \mathrm{WU}^{3}$ and AIRONG SUN ${ }^{2}$ \\ Departments of ${ }^{1}$ Pediatrics, ${ }^{2}$ Neonatology and ${ }^{3}$ Radiology, Linyi Women and Children's Hospital, \\ Linyi, Shandong 276014, P.R. China
}

Received August 29, 2018; Accepted January 23, 2019

DOI: $10.3892 / \mathrm{etm} .2019 .7305$

\begin{abstract}
This study aimed to investigate the value of MRI enhanced fluid-attenuated inversion recovery (FLAIR) combined with TNF- $\alpha$ in cerebrospinal fluid in the diagnosis of neonatal purulent meningitis. Fifty neonates with purulent meningitis were randomly selected in the purulency group, 50 patients with viral meningitis (VM) in the virus group and 50 neonates without purulent meningitis in the no meningitis group. All neonates were selected from April 2015 to May 2018 in Women and Children's Hospital (Linyi, China). Clinical baseline data of the three groups were compared, and logistic regression analysis was conducted to analyze the risk factors of the disease. The risk factors of neonatal purulent meningitis include white bold cell count, TNF- $\alpha$, encephaledema, encephaledema combined with abnormal EEG, erythrocyte sedimentation rate and FLAIR sequential apparent diffusion coefficient (ADC). TNF- $\alpha$ in cerebrospinal fluid combined with FLAIR sequential ADC obtained higher sensitivity, specificity, positive predictive value, negative predictive value, diagnostic accuracy and AUC than single TNF- $\alpha$ in cerebrospinal fluid examination or single FLAIR sequence. MRI enhanced FLAIR sequence scan combined with $\mathrm{TNF}-\alpha$ in cerebrospinal fluid has the highest rate in early diagnosis of neonatal purulent meningitis, and it is worthy of clinical promotion.
\end{abstract}

Correspondence to: Dr Xiaolin Sun, Department of Neonatology, Linyi Women and Children's Hospital, 1 Qinghe South Road, Linyi, Shandong 276014, P.R. China

E-mail: as895q@163.com

Dr Yanli Zhang, Department of Pediatrics, Linyi Women and Children's Hospital, 1 Qinghe South Road, Linyi, Shandong 276014, P.R. China

E-mail: xundandan@126.com

${ }^{*}$ Contributed equally

Key words: meningitis, suppurative, cerebrospinal fluid TNF- $\alpha$, FLAIR sequence scan

\section{Introduction}

Neonatal purulent meningitis has been given closer attention because of its high mortality rate in neonatal period and high disability rate in childhood. Moreover, the incidence rate of neonatal purulent meningitis is increased year by year due to the inappropriate use of antibiotics and increase of drug-resistance bacteria. Its incidence rate is $0.02-0.1 \%$ in live births and $0.3 \%$ in premature infants. In addition, its mortality rate is as high as $40-58 \%$, and $40-50 \%$ of survivors suffer from neurological sequelae (1). Furthermore, the symptoms of neonatal purulent meningitis are not typical, and the indiscriminate use of antibiotics can lead to atypical changes in cerebrospinal fluid (CSF), thus making the early diagnosis of neonatal purulent meningitis difficult. Therefore, exploring an effective detection method for early diagnosis of neonatal purulent meningitis is of important clinical significance. Currently, it is considered that TNF- $\alpha$ is an important cytokine participating in the inflammatory response, its level is elevated in CSF of neonates with purulent meningitis (2). With the improvement in technology, the fluid-attenuated inversion recovery (FLAIR) sequence has shown a good value in the differential diagnosis of lesions in the meninges and brain parenchyma, which can selectively inhibit CSF and obtain high-resolution T2 contrast images at the same time (3). The application of CSF TNF- $\alpha$ detection alone or magnetic resonance imaging (MRI) enhanced FLAIR sequence scanning alone in purulent meningitis has been reported $(4,5)$. In this study, the diagnostic value of MRI enhanced FLAIR sequence scanning combined with CSF TNF- $\alpha$ detection in neonatal purulent meningitis was investigated, so as to improve the accuracy rate of diagnosis of neonatal purulent meningitis, providing a basis for early treatment in clinical practice.

\section{Patients and methods}

General data. Neonates with fever and sepsis admitted to the neonatal intensive care unit (NICU) of Linyi Women and Children's Hospital (Linyi, China) from April 2015 to May 2018 received CSF routine and biochemical examinations. Fifty neonates with purulent meningitis were randomly selected as the purulency group, and 50 neonates with viral 
meningitis (VM) were selected as the virus group. Fifty neonates without purulent meningitis were selected as the no meningitis group. The neonates were 0-28 days old. There were 37 boys and 13 girls in the purulency group, 33 boys and 17 girls in the virus group, and 35 boys and 15 girls in the no meningitis group. No significant differences were found in age and sex among the three groups $(\mathrm{P}>0.05)$, and they were comparable. Diagnostic criteria: i) Neonates conforming to the diagnostic criteria for purulent meningitis (referred to the fourth edition of Practical Neonatology) (6), with common clinical symptoms including fever (sustainable or transient), vomiting, irritability and sleepiness accompanied by disturbance of consciousness, ii) neonates with bacteria found in the culture of CSF or positive CSF in smear test, and iii) those with no bacteria detected in the culture of CSF or negative smear test result, but with typical CSF changes, turbid appearance, significantly increased white blood and obviously elevated protein. Diagnostic criteria for VM (referred to the seventh edition of Textbook of Pediatrics) (7): i) Neonates diagnosed with VM according to the characteristics of neonates' electroencephalogram (EEG) and MRI images and serological examination, and ii) those with no bacteria detected in the culture of CSF or negative smear test result, no overt increase in cell count, and slight increase in protein level. Exclusion criteria: i) Premature infants and low birth weight infants, ii) neonates with intracranial hemorrhage complicated with purulent infection, iii) neonates with congenital nervous system malformations, or iv) those with congenital genetic metabolic diseases. Signed informed consents were obtained from the parents of neonates. The examinations of CSF routine, biochemistry and culture, TNF- $\alpha$ detection and head MRI FLAIR sequence examination were carried out at the early stage of the disease. This study was approved by the Ethics Committee of Linyi Women and Children's Hospital.

\section{Examination methods}

Specimen collection. CSF was taken from neonates via lumbar puncture, followed by routine examination (biochemistry and cytology). Peripheral venous blood $(5 \mathrm{ml})$ was collected and centrifuged at $1,500 \mathrm{x} g$ at room temperature for $15 \mathrm{~min}$. Then the supernatant was collected and stored at $-80^{\circ} \mathrm{C}$.

Measurement of TNF- $\alpha$ in CSF. Double-antibody enzyme-linked immunosorbent assay (ELISA) was applied. After one freeze-thaw cycle, specimens were measured by a specially-assigned individual according to the manufacturer's instructions provided by the Academy of Military Medical Sciences.

Scanning parameters and scanning method of MRI FLAIR sequence scanning. A Philips Achieva 1.5T superconducting magnetic resonance imager was used. Scanning parameters are as follows: field of view $(F O V)=24 \mathrm{~cm} \mathrm{x} 24 \mathrm{~cm}$, layer thickness $=5 \mathrm{~mm}$, interval $=1.7 \mathrm{~mm}$, TR8500ms, TE92ms, TI2400ms, and the acquisition time was $150 \mathrm{sec}$. An 8-channel Sense head coil was adopted. The scanning should be done with neonates in a quiet state. If necessary, $6 \%$ chloral hydrate was taken orally (at a dose of $1.5 \mathrm{ml} / \mathrm{kg}$ ) half an hour before the examination. Both MRI plain scan and enhanced MRI scan were required. The plain scan included the spin echo sequence FLAIR of the cross section and the lateral axis. For the enhanced scan, gadopentetate dimeglumine (Gd-DTPA) was injected first via the cubital veins at a dose of $0.1 \mathrm{mmol} / \mathrm{kg}$. The cross-sectional fast FLAIR sequence scanning was immediately performed to ensure that the scanning conditions of the FLAIR sequence were consistent.

Evaluation and analysis of scanned images. The MRI images of neonates were independently evaluated by two magnetic resonance physicians with rich clinical experience using the 'double-blind method'. If the two physicians had different evaluations, a third physician would be selected for evaluation. The FLAIR and enhanced FLAIR of the head of neonates were analyzed and evaluated based on the location and number of purulent meningitis. The enhancement, extent and quantity of cranial nerve, meningeal blood vessel, CSF and adjacent brain parenchyma were evaluated. $(-),(+)$ and $(++)$ indicated negative, positive and strongly positive, respectively, which were used to describe the signal intensity of purulent meningitis. The total positive rate $=$ (number of positive cases + number of strongly positive cases) / total number of cases. Apparent diffusion coefficient (ADC) images and ADC values were generated automatically by computer software. The ADC value was the average of 3 measurements.

Statistical analysis. Statistical Product and Service Solutions (SPSS) 17.0 software (SPSS, Inc., Chicago, IL, USA) was used for data analyses. Measurement data were expressed as mean \pm standard deviation (mean \pm SD), and t-test was employed. $\chi^{2}$ test was applied for enumeration data. Enumeration data not conforming to normal distribution could be converted into measurement data based on reality. Factors with reference value in the univariate analysis were subjected to further logistic regression analysis, so as to determine the risk factors for purulent meningitis. ANOVA was used for multiple comparisons and Least Significant Difference was the post-hoc test. $\mathrm{P}<0.05$ suggested that the difference was statistically significant.

\section{Results}

Comparisons of clinical data and biochemical indicators of neonates in the three groups. As to clinical symptoms of neonates, there were no statistically significant differences in fever [100\% (50/50), 100\% (50/50), 100\% (50/50)], convulsive seizure [33 (66.00\%), $30(60.00 \%), 15(30.00 \%)]$, sleepiness with low spirits [45 (90.00\%), $41(82.00 \%), 36(72.00 \%)]$, jaundice [38 (76\%), $12(24.00 \%), 26(52.00 \%)]$, irritability [33, (66.00\%), $25(50.00 \%), 19(38.00 \%)]$ and dyspnea [18 (20.00\%), 13 (26.00\%), 12 (24.00\%)] among the purulency, virus and no meningitis groups $(\mathrm{P}>0.05)$. However, the increase of TNF- $\alpha(\mathrm{P}=0.005)$, white blood cell count $(\mathrm{P}=0.032)$, erythrocyte sedimentation rate $(\mathrm{ESR})(\mathrm{P}=0.028)$, rate of cerebral edema alone $(\mathrm{P}=0.042)$ and rate of cerebral edema combined with abnormal EEG $(\mathrm{P}=0.030)$ in the purulency group were overtly higher than those in the virus and no meningitis groups (Table I).

Results of MRI enhanced FLAIR sequence scanning in the three groups. The results showed that there were 45 neonates with positive $(+,++)$ high signal intensity and 5 neonates with negative high signal intensity in MRI enhanced FLAIR sequence scanning in the purulency group, while in the virus 
Table I. Comparisons of biochemical indexes of children in the three groups.

\begin{tabular}{|c|c|c|c|c|c|c|c|c|}
\hline \multirow[b]{2}{*}{ Groups } & \multirow{2}{*}{$\begin{array}{c}\text { The increase } \\
\text { of TNF- } \alpha \\
(\mathrm{pg} / \mathrm{ml})\end{array}$} & \multicolumn{2}{|c|}{ Pandy test [n (\%)] } & \multirow[b]{2}{*}{$\operatorname{ESR}(\mathrm{mm} / \mathrm{h})$} & \multirow{2}{*}{$\begin{array}{l}\text { Positive } \\
\text { CSF } \\
\text { culture } \\
{[\mathrm{n}(\%)]}\end{array}$} & \multirow{2}{*}{$\begin{array}{l}\text { White blood } \\
\text { cell count } \\
>5 \times 10^{8} / 1 \\
{[\mathrm{n}(\%)]}\end{array}$} & \multirow{2}{*}{$\begin{array}{l}\text { Cerebral } \\
\text { edema } \\
\text { alone } \\
{[\mathrm{n}(\%)]}\end{array}$} & \multirow{2}{*}{$\begin{array}{c}\text { Cerebral edema } \\
\text { combined with } \\
\text { abnormal EEG } \\
{[\mathrm{n}(\%)]}\end{array}$} \\
\hline & & Positive & Negative & & & & & \\
\hline Purulency group & $132.58 \pm 31.72$ & 60 & 40 & $50.95 \pm 9.93$ & $35(70.00)$ & $40(80.00)$ & $37(74.00)$ & $23(46.00)$ \\
\hline Virus group & $21.65 \pm 0.31$ & 72 & 28 & $17.05 \pm 10.38$ & $20(40.00)$ & $5(10.00)$ & $12(40.00)$ & $6 \quad(20.00)$ \\
\hline No meningitis group & 0 & 0 & 100 & $12.35 \pm 6.29$ & $0 \quad(0.00)$ & $0 \quad(0.00)$ & $0 \quad(0.00)$ & $0 \quad(0.00)$ \\
\hline P-value & 0.006 & 0.074 & & 0.028 & 1.583 & 0.032 & 0.042 & 0.030 \\
\hline
\end{tabular}

Table II. Comparisons of MRI enhanced FLAIR sequence scan results between the two groups.

HVS signal intensity [n (\%)]

\begin{tabular}{lrrrrr}
\cline { 2 - 4 } Groups & $(-)$ & $(+)$ & $(++)$ & Total positive rate $(\%)$ & ADC value $\left(\mathrm{x} 10^{-5} \mathrm{~mm}^{2} / \mathrm{sec}\right)$ \\
\hline Purulency group & 4 & 19 & 27 & 92.00 & $31.19 \pm 8.01$ \\
Virus group & 15 & 20 & 15 & 70.00 & $63.48 \pm 15.42$ \\
No meningitis group & 50 & 0 & 0 & 0.00 & 0.058 \\
P-value & & & 0.042 & \\
\hline
\end{tabular}

Table III. Logistic regression analysis of risk factors for neonatal purulent meningitis.

\begin{tabular}{|c|c|c|c|c|c|c|}
\hline Category & $\beta$ & SE & Wald & Odds ratio & $\begin{array}{l}95 \% \text { confidence } \\
\text { interval }\end{array}$ & P-value \\
\hline White blood cell count & 0.481 & 0.668 & 8.845 & 2.033 & $1.617-4.875$ & 0.001 \\
\hline $\mathrm{TNF}-\alpha$ & 0.805 & 0.654 & 16.531 & 3.213 & $1.768-9.969$ & 0.001 \\
\hline Cerebral edema alone & 0.563 & 0.491 & 4.610 & 1.602 & $1.191-3.679$ & 0.041 \\
\hline $\begin{array}{l}\text { Cerebral edema complicated } \\
\text { with abnormal EEG }\end{array}$ & 0.429 & 0.391 & 5.789 & 1.964 & $1.430-5.285$ & 0.040 \\
\hline ESR & 1.336 & 0.018 & 7.587 & 1.973 & $1.235-6.031$ & 0.018 \\
\hline ADC value & -1.229 & 0.019 & 14.148 & 2.326 & $1.467-7.115$ & 0.001 \\
\hline
\end{tabular}

group, there were 35 cases of positive $(+,++)$ high signal intensity and 15 cases of negative high signal intensity. All 50 neonates in the no meningitis group had negative high signal intensity. Based on the $\chi^{2}$ test, the differences were statistically significant $(\mathrm{P}=0.042)$. The comparison of ADC value showed that there was a statistically significant difference between the purulency, virus and no meningitis groups $(\mathrm{P}=0.006)$ (Table II).

Analyses on risk factors for purulent meningitis. Logistic regression analysis was carried out, and it was found that the risk factors for purulent meningitis included increased white blood cell count, enhanced TNF- $\alpha$ value, cerebral edema alone, cerebral edema complicated with abnormal EEG, elevated ESR and decreased ADC value (Table III).

Value of CSF TNF- $\alpha$ detection combined with MRI enhanced FLAIR sequence scanning. According to the receiver

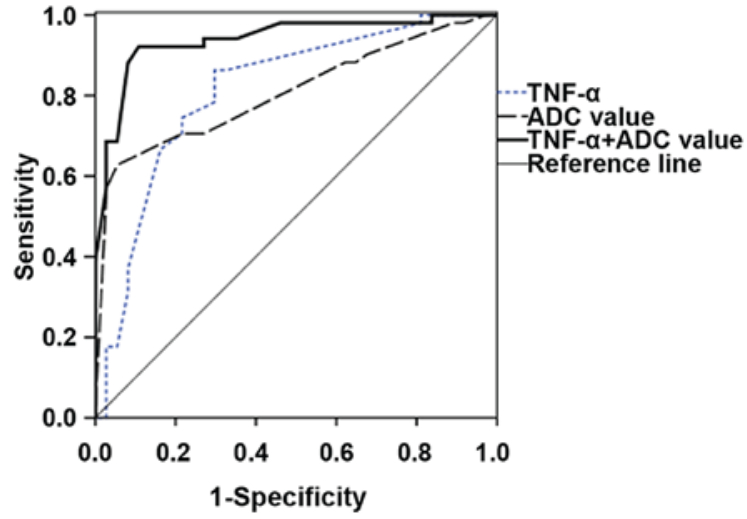

Figure 1. ROC curves of TNF- $\alpha$ and MRI enhanced FLAIR sequence ADC value in the diagnosis of neonatal purulent meningitis.

operating characteristic (ROC) curves, when the Youden index was at its maximum, the critical values of ADC in TNF- $\alpha$ 
detection and MRI enhanced FLAIR sequence examination were $74.21 \mathrm{pg} / \mathrm{ml}$ and $45.66 \times 10^{-5} \mathrm{~mm}^{2} / \mathrm{sec}$, respectively. In the measurement of ADC value, TNF- $\alpha$ detection combined with MRI enhanced FLAIR had better sensitivity, specificity, positive predictive value, negative predictive value, diagnostic accuracy and area under curve (AUC) than TNF- $\alpha$ detection alone and MRI enhanced FLAIR alone. The AUC of ROC curve showed that the the AUC of ADC value in the TNF- $\alpha$ combined detection with MRI enhanced FLAIR sequence examination was 0.925 , which was higher than those in MRI enhanced FLAIR sequence examination (0.845) and TNF- $\alpha$ detection $(0.730)(\mathrm{P}<0.05)$, showing statistically significant differences (Fig. 1 and Table IV).

\section{Discussion}

Significance and importance of TNF- $\alpha$ in CSF. Neonatal purulent meningitis is a central nervous system infection, of which the incidence rate is $0.02-0.1 \%$ in live births and up to $0.3 \%$ in premature infants. Besides, its mortality rate is $40-58 \%$, and $40-50 \%$ of survivors suffer from sequelae, such as epilepsy, hydrocephalus and mental retardation (1). For the diagnosis of purulent meningitis, routine and biochemical examinations of CSF are important means. TNF in CSF has a comprehensive biological activity, which is able to kill tumor cells, regulate $\mathrm{T}$ and $\mathrm{B}$ lymphocytes and has antisuppurative and antiviral effects (8). TNF is composed of TNF- $\alpha$ and TNF- $\beta$, in which TNF- $\alpha$ is secreted by activated monocytes and macrophages (9). Under physiological conditions, TNF in the body has a low concentration and participates in various physiological immune processes, activating neutrophils and $\mathrm{K}$ cells, promoting the growth and differentiation of B cells and stimulating the proliferation of $\mathrm{T}$ cells (10). However, the concentration of TNF is evidently increased, and its proportional relationships with other cytokines are disordered under pathological conditions, resulting in disorder of immune regulation and participating in the development of related diseases. Therefore, TNF is a potential fibrotic factor or inflammatory mediator, which plays an important role in the acute phase and inflammatory sclerosis (11). A study has manifested that in purulent meningitis, TNF- $\alpha$ is significantly elevated in CSF of neonates and involved in the inflammatory response (4). Mediators produced by viral RNA infection and endotoxin in septic lipopolysaccharide (LPS) will stimulate immune cells and infected cells to produce TNF and interleukin-6 (12). TNF- $\alpha$ can induce the production of a variety of cytokines. When neonates suffer from acute central nervous system infection, TNF- $\alpha$ is significantly increased, resulting in fever, shock and increased vascular permeability in neonates (13). This is also consistent with the results of our present study.

MRI enhanced FLAIR sequence examination and its contribution. The images were obtained via the MRI enhanced FLAIR sequence examination. This method can eliminate the artifacts of the blood vessels, showing flow voids and fully displaying the subtle lesions (14). The FLAIR sequence can suppress the CSF signal and obtain images with higher T2-weighted degree, showing lesions not visible on enhanced T1WI. In particular, the small lesions in the early stage 
of purulent meningitis can be overtly diagnosed using the enhanced FLAIR technique, to better and more timely assess the disease. Moreover, a wider range of effective information with high accuracy can be obtained through such a technique, which cannot only reflect the enhanced form and characteristics of lesions in the meninges, but also better reflect the grade and extent of lesions. In this study, the MRI enhanced FLAIR sequence scanning combined with CSF TNF- $\alpha$ detection was applied, and the results showed that its sensitivity, specificity, positive predictive value, negative predictive value, diagnostic accuracy and AUC were superior to those of TNF- $\alpha$ detection alone and MRI enhanced FLAIR sequence ADC value measurement alone.

In conclusion, our study demonstrated that the MRI enhanced FLAIR technique combined with CSF TNF- $\alpha$ detection has the highest sensitivity and specificity in the diagnosis of purulent meningitis. Therefore, it is proposed to use the MRI enhanced FLAIR technique combined with CSF TNF- $\alpha$ detection in the diagnosis of purulent meningitis, helping to improve the clinical diagnosis rate of neonatal purulent meningitis.

\section{Acknowledgements}

Not applicable.

\section{Funding}

This study was supported by Linyi Key Research and Development Project (no. 2016ZK003).

\section{Availability of data and materials}

The datasets used and/or analyzed during the current study are available from the corresponding author on reasonable request.

\section{Authors' contributions}

YZ conceived the study and drafted the manuscript. YZ and XS performed and analyzed MRI FLAIR sequence scanning. JW analyzed the general data of patients and revised the manuscript. AS helped with ELISA. All authors read and approved the final manuscript.

\section{Ethics approval and consent to participate}

This study was approved by the Ethics Committee of Linyi Women and Children's Hospital (Linyi, China). Patients who participated in this study had complete clinical data. Signed informed consents were obtained from the parents of the patients.

\section{Patient consent for publication}

Not applicable.

\section{Competing interests}

The authors declare that they have no competing interests.

\section{References}

1. The Collaborative Group For Neonatal Meningitis Study TC and Liu CQ: Epidemiology of neonatal purulent meningitis in Hebei Province, China: A multicenter study. Zhongguo Dang Dai Er Ke Za Zhi 17: 419-424, 2015 (In Chinese).

2. Prasad R, Kapoor R, Srivastava R, Mishra OP and Singh TB: Cerebrospinal fluid TNF- $\alpha$, IL-6, and IL-8 in children with bacterial meningitis. Pediatr Neurol 50: 60-65, 2014.

3. Jeevanandham B, Kalyanpur T, Gupta $\mathrm{P}$ and Cherian M: Comparison of post-contrast 3D-T1-MPRAGE, 3D-T1-SPACE and 3D-T2-FLAIR MR images in evaluation of meningeal abnormalities at 3-T MRI. Br J Radiol 90: 20160834, 2017.

4. Pfausler B, Grubwieser G, Bösch S, Vollert H, Herald M and Schmutzhard E: Cerebrospinal fluid-filtration reduces TNF alpha in bacterial meningitis-CSF. Eur J Neurol 2: 570-572, 1995.

5. Abe M, Takayama Y, Yamashita H, Noguchi M and Sagoh T: Purulent meningitis with unusual diffusion-weighted MRI findings. Eur J Radiol 44: 1-4, 2002.

6. Sohn CH, Sevick RJ, Frayne R, Chang HW, Kim SP and Kim DK: Fluid attenuated inversion recovery (FLAIR) imaging of the normal brain: Comparisons between under the conditions of 3.0 Tesla and 1.5 Tesla. Korean J Radiol 11: 19-24, 2010.

7. Nigrovic LE, Fine AM, Monuteaux MC, Shah SS and Neuman MI: Trends in the management of viral meningitis at United States children's hospitals. Pediatrics 131: 670-676, 2013.

8. Bociąga-Jasik M, Garlicki A, Cieśla A, Kalinowska-Nowak A, Sobczyk-Krupiarz I and Mach T: The diagnostic value of cytokine and nitric oxide concentrations in cerebrospinal fluid for the differential diagnosis of meningitis. Adv Med Sci 57: 142-147, 2012.

9. Panato AP, Tomasi LT, Simon CS, Madeira K, Simoes LR, Medeiros LR, Barichello T and Rosa MI: Meta-analysis identifies tumor necrosis factor-alpha and interleukin-1 beta as diagnostic biomarkers for bacterial and aseptic meningitis. Curr Neurovasc Res 11: 340-348, 2014.

10. Splendiani A, Puglielli E, De Amicis R, Necozione S, Masciocchi $C$ and Gallucci M: Contrast-enhanced FLAIR in the early diagnosis of infectious meningitis. Neuroradiology 47: 591-598, 2005.

11. Letiembre M, Echchannaoui H, Ferracin F, Rivest $S$ and Landmann R: Toll-like receptor-2 deficiency is associated with enhanced brain TNF gene expression during pneumococcal meningitis. J Neuroimmunol 168: 21-33, 2005.

12. Kim HJ, Shim KW, Lee MK, Park MS, Kim SH, Kim EY, Park S and Kim TS: Tuberculous encephalopathy without meningitis: Pathology and brain MRI findings. Eur Neurol 65: 156-159, 2011.

13. Šumanović-Glamuzina D, Čulo F, Čulo MI, Konjevoda P and Jerković-Raguž M: A comparison of blood and cerebrospinal fluid cytokines (IL-1 $\beta$, IL-6, IL-18, TNF- $\alpha$ ) in neonates with perinatal hypoxia. Bosn J Basic Med Sci 17: 203-210, 2017.

14. Vaswani AK, Nizamani WM, Ali M, Aneel G, Shahani BK and Hussain S: Diagnostic accuracy of contrast-enhanced FLAIR magnetic resonance imaging in diagnosis of meningitis correlated with CSF analysis. ISRN Radiol 2014: 578986 , 2014.

This work is licensed under a Creative Commons Attribution-NonCommercial-NoDerivatives 4.0 International (CC BY-NC-ND 4.0) License. 